\title{
Normenwandel und Justizreform - Das Beispiel der Arbeitsgerichtsbarkeit
}

Das Arbeitsrecht grenzt sich seit jeher vom bürgerlichen Recht ab. Sein Status als eigenständiger Rechtsbereich wurde historisch mit der Besonderheit der Natur des Arbeitsvertrags begründet. Vom Rest der Justiz weitgehend unabhängige Arbeitsgerichte sollten die Eigenständigkeit des materiellen Rechts institutionell absichern. Doch seit einiger Zeit werden die Arbeitsgerichte schrittweise in den Einzugsbereich der Justizverwaltung zurückgeführt. Die Liberalisierung der Sozialdemokratie seit den 1960er Jahren hat an dieser Entwicklung einen beträchtlichen Anteil.

\section{Das Normenverständnis im Arbeitsrecht}

\subsection{EIN ALTER STREIT...}

Der Normenwandel im Arbeitsrecht kann in vielen Bereichen als Liberalisierung beschrieben werden. Um dies zu verstehen, ist ein Blick auf die Ursprünge des Arbeitsrechts hilfreich. Der Sozialismus bzw. die Sozialdemokratie war historisch eine Gegenbewegung zum Liberalismus. Und das im Rahmen dieses Konflikts entstandene Arbeitsrecht entwickelte sich entlang der Kritik am Bürgerlichen Gesetzbuch, insbesondere an der dort verankerten liberalen Rechtsnorm der Vertragsfreiheit (Graf 1993, S.95). Sozialdemokratische Juristen wie Hugo Sinzheimer argumentierten auf der Basis der Marx'schen Frühschriften und des Genossenschaftsrechts von Otto von Gierke, dass die Rechtsnorm der Vertragsfreiheit auf dem Arbeitsmarkt aufgrund der strukturellen Ungleichheit von Arbeit und Kapital faktisch nicht gelte (Sinzheimer 1976 [1924]). Der Arbeitnehmer, der in seiner persönlichen Existenz vom Arbeitsverhältnis abhängig sei, habe nicht die Wahl, schlechte Verträge auszuschlagen. Nur im Kollektiv, d. h. im Rahmen organisierter Gewerkschaften, könne die Arbeitnehmerseite auf gleicher Augenhöhe mit den Unternehmen verhandeln. Deswegen sollten Gewerkschaften stellvertretend die zentralen Arbeitsbedingungen aushandeln, um den einzelnen Beschäftigten vor Selbstausbeutung zu schützen. Das zentrale Instrument sind Tarifverträge, die als unmittelbar zwingendes und unabdingbares Recht auf das individuelle Arbeitsverhältnis einwirken.
Liberale Kritiker sahen in den arbeitsrechtlichen Grundannahmen damals wie heute eine Einschränkung der individuellen Vertragsfreiheit. Hugo Sinzheimer hat demgegenüber argumentiert, dass Tarifverträge die Vertragsfreiheit der Arbeitnehmer nicht einschränken, sondern ermöglichen würden. Erst wenn unabdingbare Mindeststandards tarifvertraglich abgesichert sind, können freie Verträge geschlossen werden. Das Arbeitsrecht reklamiert für sich einen Status als Sonderrecht, also als ein Recht, das nicht unter das allgemeine Vertragsrecht subsumiert werden soll.

Die Auseinandersetzung zwischen den liberalen und den sozialdemokratischen Kräften um den Sonderstatus des Arbeitsrechts muss auch im historischen Kontext betrachtet werden. Mit dem Bürgerlichen Gesetzbuch hatte das Deutsche Reich erstmals zu einer einheitlichen Rechtsordnung gefunden. Diese sollte nach liberalen Vorstellungen nicht gleich wieder durch einen neuen ,Separatismus “ des Arbeitsrechts infrage gestellt werden (Graf 1993, S. 138). Zudem galt das Recht des Individuums, freie Verträge ohne die Einwirkung staatlichen Zwangs zu schließen, als die zentrale Errungenschaft der bürgerlichen Gesellschaft. Demgegenüber machten die Sozialdemokraten die Verelendung der Arbeiter in der kapitalistischen Ökonomie geltend. Das Proletariat profitiere nicht von der Freiheit des Vertragsrechts, sondern leide unter dem Zwang des Marktes und der Herrschaft der Unternehmer, die sie unter dem Deckmantel scheinbar freiwillig geschlossener Verträge ausbeuteten.

\section{$1.2 \ldots$ NEU AUFGELEGT}

Wenn heute ein Prozess der Liberalisierung stattfindet, ist damit gemeint, dass der Sonderstatus des Arbeitsrechts schrittweise zurückgenommen wird. Der Wandel bedeutet also nicht, dass neue Normen an Bedeutung gewinnen. Er vollzieht sich vielmehr als eine Renaissance historisch bekannter Ideen. Arbeitsverträge werden anderen Verträgen (wieder) häufiger gleichgestellt. Der Regulierungsvorrang der Tarifparteien sowie die zwingende Wirkung von Tarifverträgen wurden in den vergangenen 20 Jahren teilweise relativiert, und Arbeitnehmer sind heute auf betrieblicher Ebene häufiger mit der Aufgabe konfrontiert, ihre Arbeitsbedingungen (freiwillig oder unfreiwillig) partiell selbst neu auszuhandeln und ihre Vertragsfreiheit auch ohne tarifliche Grundabsicherung wahrzunehmen. Dieser Prozess lässt sich in allen Arenen der industriellen Beziehungen beobachten.

Auf betrieblicher Ebene sind Forderungen nach flexiblen, meist marktangepassten tarifrechtlichen Regelungen seit Mitte der 1980er Jahre kontinuierlich lauter geworden. Sie reagieren unter anderem auf veränderte Marktbedingungen und einen national wie international gestiegenen Wettbewerbsdruck. Betriebliche Bündnisse für Arbeit, die Beschäftigungssicherheit im Tausch gegen tarifrechtliche Konzessionen der Arbeitnehmer versprechen, bringen diesen Wandel zum Ausdruck (MassaWith/ Seifert 2004; Rehder 2003). Gewerkschaften und Arbeitgeberverbände haben den schleichenden Liberalisierungsprozess auf der tariflichen Regulierungsebene teils zögerlich und teils aktiv mitgestaltet

Britta Rehder, Dr., ist wissenschaftliche Mitarbeiterin am Max-Planck-Institut für Gesellschaftsforschung. Arbeitsschwerpunkte: Industrielle Beziehungen, Politik und Recht. e-mail: rehder@mpifg.de 
(Bispinck 2004). Die Etablierung von Öffnungsklauseln als Flexibilisierungsinstrument nimmt Tarifverträgen einen Teil ihres zwingenden Charakters. Die Möglichkeiten, die Wirkungsrichtung von Tarifverträgen durch betriebliche Vereinbarungen nachzujustieren, sind zahlreich geworden.

Diese Entwicklung wurde auch von der Politik gefördert. Jahrzehntelang waren die großen Parteien mit Vorschlägen zur Ausgestaltung des Tarifrechts zurückhaltend gewesen. Die verfassungsrechtlich verankerte Koalitionsfreiheit machte Reformvorschläge in diesem Politikfeld tendenziell aussichtslos und wahlpolitisch risikoreich. Doch die Zurückhaltung wird seit Ende der 1990er Jahre schrittweise aufgegeben. Die SPD hat unter der Kanzlerschaft von Gerhard Schröder eine Flexibilisierung der Tarifpolitik gefordert. Dies gilt noch ausgeprägter für die CDU/CSU, die mit Plänen für eine liberale Reform des Arbeitsrechts in den Bundestagswahlkampf 2005 zog (Rehder 2006, S.189).

Und schließlich lassen sich Liberalisierungstendenzen auch im Rechtssystem beobachten. Eine Interpretation des arbeitsrechtlichen Günstigkeitsprinzips, die es den Betrieben freistellen will, selbst zu entscheiden, ob die geltenden Tarifverträge oder ausgehandelte betriebliche Abweichungen für die Beschäftigten günstiger sind, etablierte sich seit den 1980er Jahren im arbeitsrechtswissenschaftlichen Diskurs und wurde erst vom Ersten Senat des Bundesarbeitsgerichts gestoppt (ebd.). Auch innerhalb des Bundesarbeitsgerichts gab es Befürworter der Neuinterpretation des Günstigkeitsprinzips. Der Vorsitzende des Vierten Senats und heutige Justizminister Thüringens, Harald Schliemann, hatte das Urteil mehrmals öffentlich infrage gestellt.

\section{Normenwandel und Arbeitsgerichte}

Die Arbeitsgerichtsbarkeit selbst ist ein selten beachtetes Beispiel für den Normenwandel. Hier finden Transformationsprozesse statt, die die Liberalisierung des Arbeitsrechts institutionell verfestigen, denn Institutionen symbolisieren Normen. Die Einrichtung selbstständiger Arbeitsgerichte im Jahr 1926 sollte den Sonderstatus des Arbeitsrechts zementieren und ihm eine institutionelle Heimat geben: Weitgehend unabhängig vom Justizapparat und unter der Obhut der Arbeitsministerien sollten Gerichte mit speziell qualifizierten Richtern arbeitsrechtliche Streitigkeiten beilegen. Der Sonderstatus der Gerichte symbolisierte den Sonderstatus des Arbeitsvertrags. Doch ihre Unabhängigkeit blieb fragil, sie wurde immer wieder infrage gestellt. Kleine und fortschreitende Justizreformen haben das ihrige getan: Sie haben die Eigenständigkeit der Arbeitsgerichte teilweise zurückgenommen.

Diese Entwicklung ist nicht allein das Resultat erfolgreicher neoliberaler Strategien. Auch wenn die Justiz und die FDP historisch kaum eine Gelegenheit ausgelassen haben, um die Eigenständigkeit der Arbeitsgerichte zur Disposition zu stellen, wären sie allein kaum dazu in der Lage gewesen, etwas zu bewirken. Aber der Prozess wurde in eigentümlicher Weise von der SPD mit (und zuweilen entscheidend) vorangetrieben - manchmal aus Überzeugung, manchmal aufgrund konfligierender Zielvorstellungen oder mangelnder Weitsicht.

Justizreformen sind nicht nur das Ergebnis, sondern gleichzeitig potenzielle Ursache der Liberalisierung, denn Form und Inhalt beeinflussen sich wechselseitig. Wenn die „Produktionsbedingungen der Rechtsprechung" verändert werden, hat dies mittelfristig auch Folgen für die Urteilsbildung. Dies gilt insbesondere im Arbeitsrecht, das Richterrecht ist. So haben die Arbeitsgerichte zum Beispiel das Streikrecht geprägt. Sie urteilen darüber, ob eine Arbeitnehmergruppierung den Status als Gewerkschaft für sich beanspruchen kann und welche Strategien zulässig sind. Das heißt, sie konstituieren Akteure und deren Machtressourcen.

Die US-amerikanische Forschung über Gerichte hat gezeigt, dass schon geringfügige Veränderungen der institutionellen Umwelt den Gang der Rechtsprechung beeinflussen können. So hat im U. S.-Supreme Court die Praxis der Richter, ein Urteil einstimmig zu fällen, rapide an Bedeutung verloren, nachdem das Gericht seinen Amtssitz verlagerte. Bis 1935 hatte das oberste Bundesgericht der Vereinigten Staaten im Capitol residiert. Mit dem Umzug in ein eigenes Gebäude wuchs die Heterogenität zwischen den Richtern und deren Bereitschaft, individuell abweichende Stellungnahmen in Ergänzung zum Gerichtsurteil zu veröffentlichen. Das neue Gebäude hatte es ermöglicht, verbesserte technische Arbeitsbedingungen (erweiterte
Bibliothek etc.) einzurichten und wissenschaftliche Assistenten zu beschäftigen, wodurch abweichende und differenzierte Urteile möglich wurden. Im weiteren Verlauf entwickelten sich diese abweichenden Stellungnahmen teilweise als Ausgangspunkt für neue rechtswissenschaftliche und rechtspolitische Entwicklungen (O'Brien 1999, S. 102).

Vor diesem Hintergrund ist es lohnend, die Transformationsprozesse in der Arbeitsgerichtsbarkeit in den Blick zu nehmen. Die folgende Analyse reicht historisch zurück in die Weimarer Republik, denn in dieser Phase wurden viele Diskussionen geführt, die uns heute sehr bekannt vorkommen.

\section{Die Situation in der Weimarer Republik}

Das Arbeitsrecht zählt zum wichtigen politischen Erbe der Weimarer Republik. Zentrale Gesetze und Institutionen, die seinerzeit etabliert wurden, sind auch heute noch Bestandteil unseres Rechtssystems. Das Arbeitsgerichtsgesetz wurde im Jahr 1926 verabschiedet. Die Ausgestaltung der Arbeitsgerichtsbarkeit hatte in der politischen Debatte der 1920er Jahre einen sehr hohen Stellenwert (Kraushaar 1987). Die Organisationsfrage wurde in einem unmittelbaren und symbolträchtigen Zusammenhang mit dem Status des materiellen Rechts gesehen.

\subsection{UMSTRITTENER STATUS DER ARBEITSGERICHTBARKEIT}

Die Befürworter des Arbeitsrechts als Sonderrecht argumentierten regelmäßig auch für die Einrichtung eigenständiger Arbeitsgerichte als institutionelle Absicherung. Das materielle Recht sollte durch die Interpretation und Fortbildung im Rahmen einer speziellen Gerichtsbarkeit in eine eigene Richtung (sprich: weg vom Vertragsrecht) weiterentwickelt werden. Die Beteiligung von ehrenamtlichen Richtern sollte die Praxisnähe der Rechtsprechung sichern und die sich schnell wandelnden betrieblichen Produktions- und Arbeitsbedingungen reflektieren. Das beschleunigte Verfahren sollte der existenziellen Bedeutung des Arbeitsverhältnisses für die Beschäftigten gerecht werden (Opolony 2004, S. 523). 
Kurzum: Materielles Arbeitsrecht und selbstständiges Arbeitsgericht galten als eine Kompensation für die Marktmacht des Unternehmers.

Die stärksten Befürworter dieser Regelung waren die freien Gewerkschaften und die ihnen nahestehenden Juristen wie $\mathrm{Hu}$ go Sinzheimer (Weiß 1994, S. 123). Die Motivation der Gewerkschaften lag dabei auch in ihrem ausgeprägten Misstrauen gegenüber der Justiz (Bähr 1991, S. 512). Die meisten Richter der Weimarer Republik hatten schon in der Kaiserzeit Recht gesprochen und standen im Verdacht der „Klassenjustiz“. Viele waren aktiv an der Verfolgung von Sozialdemokraten und Gewerkschaftern beteiligt gewesen. Und das Reichsgericht hatte erst im Jahr 1910 erstmalig die verbindliche Wirkung von Tarifverträgen anerkannt - nach mehr als 40 Jahren tarifpolitischer Praxis (Blanke et al. 1975, S. 129). Das Arbeitsrecht sollte, gänzlich entkoppelt vom Justizapparat, implementiert und ausgestaltet werden. Die Justiz und ihre Berufsverbände waren dementsprechend die stärksten Gegner eigenständiger Arbeitsgerichte (Graf 1993, S. 159). Auch der Deutsche Juristentag sprach sich entschieden gegen die Abspaltung von der ordentlichen Gerichtsbarkeit aus. Er wurde dabei teilweise von Arbeitgeberseite und der Deutsch-Nationalen Volkspartei unterstützt.

Zwischen den beiden Extrempolen gab es andere Positionen, die facettenreiche Abstufungen der arbeitsgerichtlichen Unabhängigkeit vorschlugen. Prominent wurde dabei vor allem die Unterscheidung zwischen „eingegliederten “ und ,angegliederten "Arbeitsgerichten. In beiden Konzepten sollten die Arbeitsgerichte nicht unabhängig sein, sondern Abteilungen der ordentlichen Gerichte werden. In den „eingegliederten Arbeitsgerichten" sollte jedoch der Vorsitzende aus dem Kreis der ordentlichen Richter gewählt werden, während bei den ,angegliederten“ Gerichten lediglich die Befähigung zum Richteramt genügen sollte. Damit hätten auch gewerkschaftliche Juristen ernannt werden können. Die Arbeitsgerichte wären dann institutionell an die Justiz angebunden worden, personell jedoch vom Berufsstand der Richter entkoppelt gewesen (Bohle 1990, S. 92).

Gestritten wurde auch über die fachliche Zuordnung der neuen Gerichtsbarkeit in die Ressortzuständigkeiten. Die Befürworter unabhängiger Arbeitsgerichte plädierten für eine Dienstaufsicht und Ver- waltung durch das Arbeitsministerium. Die Gegner forderten eine Zuteilung zum Justizressort. Zwischenpositionen schlugen eine geteilte Verantwortlichkeit mit unterschiedlichen Vorgriffsrechten des einen oder anderen Ministeriums vor. Es gab viele weitere strittige Organisationsfragen, die alle ein gemeinsames Merkmal hatten: Immer wurde die Organisationsfrage verknüpft mit der materiellen Frage, ob die jeweils vorgeschlagene Organisationsform die Unabhängigkeit der Arbeitsgerichte (und damit den Charakter des Arbeitsrechts als eigenständiger Rechtsbereich) vergrößern oder schmälern würde.

Jenseits der Deutsch-Nationalen Volkspartei, die das Lager der Kritiker an eigenständigen Gerichten unterstützte, verlief die parlamentarische Konfliktlinie eher quer zu (statt entlang von) Parteizugehörigkeiten. Bis weit ins bürgerliche Lager hinein (Zentrum, Deutsche Demokratische Partei) wurde das Interesse der Gewerkschaften an einer unabhängigen $\mathrm{Ge}$ richtsbarkeit geteilt oder zumindest ernst genommen. Gleichzeitig hat sich die SPD nicht geschlossen für eine Separierung ausgesprochen. Der sozialdemokratische Justizminister Gustav Radbruch argumentierte, die Ausgliederung der Arbeitsgerichte hätte zur Folge, dass die ordentliche Justiz mit den Arbeitern nur noch als Strafgerichte befasst wäre:

„Die Ausgliederung der Arbeitsgerichte aus der Justiz würde nach meiner Auffassung das Todesurteil über unsere ordentliche Justiz bedeuten. Es würde bedeuten, dass unsere ordentliche Justiz wirklich zu einer Klassenjustiz in einem freilich anderen Sinne herabsänke. Dann würde alle soziale Justiz von ihr abgenommen sein, und es bliebe nur die Bourgeoisjustiz über kapitalistische Rechtsstreitigkeiten übrig" (zitiert nach Graf 1993, S. 195).

Vor diesem Hintergrund überlagerten Ressortstreitigkeiten zwischen den Fachministerien die parteipolitischen Lager. Das Arbeitsministerium plädierte mit der Unterstützung des von der Weimarer Koalition eingesetzten Arbeitsrechtsausschusses - auch unter dem Druck der Gewerkschaften - für eine relativ starke Unabhängigkeit der Gerichte, während das Justizministerium - angetrieben durch die Opposition der Justiz - für eine mehr oder weniger starke Eingliederung in die ordentliche Justiz arbeitete.

\subsection{ANGLIEDERUNG AN DIE JUSTIZ}

In dieser Konfliktkonstellation wurden die Finanzministerien in Bund und Ländern sowie kommunalpolitische Akteure, wie z. B. der Deutsche Städtetag, zum Zünglein an der Waage (Bohle 1990, S. 96). Aufgrund ihrer Sorge über die Kosten eines zusätzlichen Gerichtszweigs unterstützten sie die Position der Justiz und der Berufsverbände. In dem Maße, wie sich die wirtschaftliche Situation in der Weimarer Republik zusehends verschlechterte, sanken die Chancen auf die Durchsetzbarkeit gänzlich unabhängiger Arbeitsgerichte. Das Arbeitsministerium konnte sich demgegenüber kaum durchsetzen und schloss sich schließlich der Position des Justizministeriums mehr oder weniger an. So sah das letztlich verabschiedete Arbeitsgerichtsgesetz nur in der ersten Instanz selbstständige Arbeitsgerichte vor. Die Landesarbeitsgerichte sowie das Reichsarbeitsgericht wurden hingegen in die ordentliche Justiz eingegliedert. Die Einrichtung, Verwaltung und Dienstaufsicht übernahm das Justizministerium. Das Vorhaben, die Etablierung des Arbeitsrechts als Sonderrecht durch ein gegen die Justiz abgeschirmtes Gerichtswesen institutionell abzusichern, war nur teilweise gelungen. Die fiskalische Argumentation hatte sich durchgesetzt.

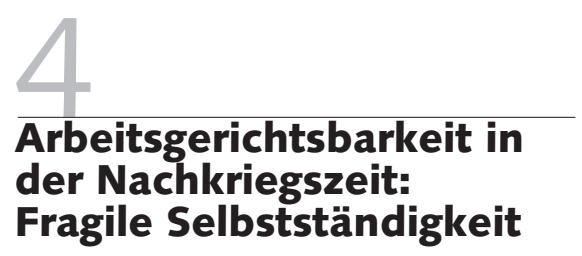

Nach dem Ende des Zweiten Weltkriegs lag eine gänzlich veränderte Konstellation vor. Zwar ordneten die amerikanischen Militärbehörden 1945 die Schließung der Arbeitsgerichte an (Neumann 1993, S. 343). Diese Position wurde aber wenige Monate später revidiert (Steidle 1946, S.14). Das Kontrollratsgesetz Nr. 21 etablierte selbstständige Arbeits- und Berufungsgerichte, die den Provinz- oder Landesarbeitsbehörden unterstellt wurden. Berufsrichter waren nicht zwingend vorgesehen. Die Vorsitzenden mussten lediglich die „besondere Befähigung in Arbeitsangelegenheiten " vorweisen. Damit waren die gewerkschaftlichen Forderungen aus der Weimarer Republik erfüllt.

Die rechtshistorische Literatur gibt kaum Auskunft darüber, wie diese für die 
gesamte Nachkriegsperiode relevante Entscheidung zustande kam. Doch lässt sich vermuten, dass die Gewerkschaften aus verschiedenen Gründen gegenüber ihren Gegenspielern einen erheblichen Einflussvorsprung hatten. Die amerikanische Regierung hatte schon 1944 begonnen, die Besetzung Deutschlands und die Arbeit einer Militärregierung vorzubereiten. Dabei zählte sie auf die Gewerkschaften als starke gesellschaftliche Kraft. Und sie sah in den Arbeitnehmerverbänden eine Quelle des Widerstands gegen den Nationalsozialismus (Nautz 1985, S. 42). Außerdem ließ sich die US-Regierung bei ihren Vorbereitungen von deutschen Emigranten beraten, die vor dem Nazi-Regime geflüchtet waren. Für den Bereich des Arbeitsrechts griff sie auf die Unterstützung von Franz Neumann zurück (ebd., S. 43). Dieser war in der Weimarer Zeit ein Schüler von Hugo Sinzheimer gewesen, der stark für die Selbstständigkeit der Arbeitsgerichte plädiert hatte. Darüber hinaus dürfte die US-Regierung auch besonders empfänglich für die gewerkschaftliche Forderung nach einer eigenständigen Gerichtsbarkeit gewesen sein. Sie selbst hatte in der „New Deal“-Ära der 1930er Jahre die Erfahrung gemacht, wie schwer es war, sozialpolitische Programme gegen den konservativ eingestellten Supreme Court durchzusetzen (O'Brien 1999, S. 95).

Das Grundgesetz etablierte 1948/49 ein selbstständiges Arbeitsgericht auch auf Bundesebene. Der Entwurf des Verfassungskonvents von Herrenchiemsee hatte noch ein in die ordentliche Gerichtsbarkeit eingegliedertes Arbeitsgericht vorgesehen. Doch die Sozialdemokraten und Gewerkschaften konnten sich in den Beratungen des Parlamentarischen Rats durchsetzen, insbesondere gegen den Widerspruch der FDP. Der Erfolg lag nicht zuletzt auch daran, dass den Gegnern einer Aufspaltung des Justizapparats die Einrichtung eines obersten Gerichtshofes in Aussicht gestellt wurde, der dann die verschiedenen Fachrichtungen wieder zusammenführen würde. Doch dieses Konzept ist nie realisiert worden. Stattdessen wurde zur Wahrung der einheitlichen Rechtsprechung ein Gemeinsamer Senat eingerichtet, der sich aus Vertretern der obersten Fachgerichte zusammensetzt (Bonner Kommentar 1950).

Damit war die Unabhängigkeit der Arbeitsgerichtsbarkeit auf ihrem Zenit angelangt. Doch gleichzeitig begannen die Arbeiten an ihrer partiellen Rückführung in die Obhut der Justiz. Im Vorlauf der Beratungen zum Arbeitsgerichtsgesetz (1953), das die Vorgaben des Grundgesetzes realisieren und die Arbeitsgerichtsbarkeit auf eine integrierte gesetzliche Grundlage stellen sollte, formierten sich erneut Gegner und Befürworter (Kraushaar 1987, S. 2311). Die Schwerpunkte der Diskussion verlagerten sich. Konnte die Selbstständigkeit der Arbeitsgerichte durch die Regelung des Grundgesetzes nun (vorläufig) nicht mehr verhindert werden, konzentrierten sich die Gegner auf die Ressortzugehörigkeit und die Richterrekrutierung, um die Anbindung an den Justizapparat zu halten. Auseinandersetzungen zwischen Bund- und Länderebene machten die Einsetzung eines Vermittlungsausschusses notwendig (Leinemann 1991, S. 966). Einige Länder hatten in den Jahren 1946/47 auf der Basis des Kontrollgesetzes Nr. 21 bereits eigene Arbeitsgerichtsgesetze erlassen (z. B. Hessen). Der Entwurf der Bundesregierung sah demgegenüber vor, die Dienstaufsicht der Arbeitsbehörden in wesentlichen Geschäften an das Einvernehmen der Justizverwaltung zu binden (Neumann 1993, S. 343). Zudem sah er vor (entgegen den Bestimmungen des Kontrollratsgesetzes), dass nur Volljuristen zugelassen werden.

Das heißt: Der Gesetzentwurf sah eine stärkere Unabhängigkeit von der Justiz vor als das Arbeitsgerichtsgesetz von 1926. Er war aus Sicht der Gewerkschaften jedoch ein Rückschritt gegenüber den Nachkriegsregelungen bzw. einigen Landesgesetzen. Diese Entwicklung dürfte mit der Regierungsbeteiligung der FDP auf Bundesebene zusammhängen, die sich als einzige Partei in Bund und Ländern mehr oder weniger geschlossen für eine starke Eingliederung der Arbeitsgerichte ausgesprochen hatte und die zudem das Justizressort besetzte. Zumindest bei der Frage der Ressortierung setzte sich die Bundesregierung durch.

In der Folgezeit zeigte sich, dass es für eine "kleine“ Sondergerichtsbarkeit nicht einfach sein würde, die notwendigen Ressourcen zur Aufrechterhaltung des Status quo sicherzustellen. Dies gilt bis heute. Ungefähr 1.000 Arbeitsrichter stehen 15.000 Richtern der ordentlichen Justiz gegenüber (Stand: 2004, Statistisches Bundesamt). Die Anreize, bei übergreifenden Fragen, wie Ausbildung oder Auswahl von Richtern, keine spezifisch arbeitsgerichtlichen Regelungen zuzulassen, waren immer hoch und stiegen mit den eventuell anfallenden Kosten von Sonderregelungen. So beklagten sich die Präsidenten der Landesarbeitsgerichte im Jahr 1961 über den Mangel an geeignetem Richternachwuchs. Sie führten dies auf ein zu geringes einschlägiges Lehrangebot an den Universitäten zurück und forderten die Einrichtung eines Lehrstuhls für Arbeitsrecht an jeder rechtswissenschaftlichen Fakultät (Nikisch 1961, S. 158). Diese Forderung wurde nicht erfüllt. Stattdessen wurde im gleichen Jahr ein Richtergesetz erlassen, das "das Bild des einheitlichen Richters“ schuf (Kern 1961, S. 618). Der Zwang zum Volljuristen, der von der Bundesregierung schon in den Verhandlungen zum Arbeitsgerichtsgesetz favorisiert worden war, wurde nun realisiert. Zudem entfielen alle Bestimmungen, die für die verschiedenen Zweige der Gerichtsbarkeit besondere Kenntnisse voraussetzten. Damit wurde der Übergang von einem Gerichtszweig zu einem anderen bzw. deren Austausch möglich. Dies wiederum senkte beim studentischen Nachwuchs die Anreize, sich speziell und zusätzlich im „kleinen“ Arbeitsrecht ausbilden zu lassen, wenn doch die generelle Qualifikation im bürgerlichen Recht auch reichen sollte. Eine arbeitsrechtliche Qualifikation erwarb nur, wer dies explizit anstrebte.

\section{Die 1960er Jahre: Diskus- sionen um die Idee des Rechtspflegeministeriums}

Unabhängig von diesen Ereignissen, aber zeitgleich und mit langfristigen Folgen für die Arbeitsgerichtsbarkeit, entwickelte sich eine andere Diskussion. Die Erfahrung mit dem Nationalsozialismus förderte die Idee, das Rechtssystem gegenüber der Politik abzuschotten und die Politik durch das Rechtssystem kontrollieren zu lassen. Schon die Nürnberger Kriegsverbrecherprozesse verdeutlichten hohe Erwartungen an die Leistungsfähigkeit von Gerichten: Ein Gericht sollte die Schuldfragen des Nationalsozialismus klären und die Schuldigen angemessen bestrafen. Zudem wurde mit dem Bundesverfassungsgericht eine Institution neu geschaffen, die als „Hüterin der Verfassung " wirken sollte und deren Aufgabe auch darin bestand, die Exekutive $\mathrm{zu}$ kontrollieren. Gleichzeitig wurde im Verlauf der Nachkriegsgeschichte (schritt- 
weise) unübersehbar, dass die Gerichte den Nationalsozialismus teilweise aktiv gestützt haben (Rüthers 1968). Deswegen wurde die Frage der richterlichen Unabhängigkeit zu einem zentralen Thema der Rechtspolitik. Deutlich wurde dies auch in den Auseinandersetzungen um das bereits erwähnte Richtergesetz, in denen das federführende Justizressort mit dem Innenministerium über die Trennung von Exekutive und Justiz stritt (Kern 1961). Strittig war in diesem Zusammenhang unter anderem die Frage, ob Richter Beamte sind oder einen unabhängigen Status für sich beanspruchen können.

Die Debatte drehte sich auch um die Schaffung eines „Rechtspflegeministeriums" für die öffentlich-rechtlichen Gerichtsbarkeiten. Die Aufsicht über die Finanz- und Verwaltungsgerichte sollte nach diesem Konzept nicht länger bei den Fachressorts angesiedelt werden, sondern in das Justizressort wandern, um eine möglichst starke Trennung von Verwaltung und Justiz zu realisieren. Auch hier verlief die Linie zwischen Anhängern und Befürwortern wieder quer durch die Parteien. Schleswig-Holstein und Hamburg realisierten das Rechtspflegeministerium schon in den 1950er Jahren: im ersten Fall unter der Führung der CDU, im zweiten unter der SPD. Die FDP hat sich sehr zeitig geschlossen auf die Forderung nach einem Rechtspflegeministerium festgelegt (Wassermann 1964, S. 256).

Im Verlauf der 1960er Jahre setzte sich diese Idee auch in der SPD stärker durch, und sie wurde zu einem Pfeiler der sozialliberalen Koalition unter Willy Brandt, allerdings mit anderer Akzentuierung als noch in der Nachkriegszeit. Ging es nach dem Zweiten Weltkrieg um die Trennung von Exekutive und Judikative zugunsten der richterlichen Unabhängigkeit, so standen in der Brandt-Ära die Rechte des Bürgers gegenüber dem Staat im Vordergrund. Die Neuressortierung der Verwaltungsund Finanzgerichte unter das Justizressort war Bestandteil des Programms „Mehr Demokratie wagen" (Brandt (1979 [1969]). Der Bürger, der sein Recht gegen die Interessen des Staats in Anspruch nehmen wollte, sollte nicht einem Gericht gegenüber treten, dessen Dienstherr gleichzeitig Angeklagter ist.

In der Folge sahen sich auch die Arbeitsgerichte mit einer alten Forderung (aber neuer Begründung) konfrontiert. Dies lässt sich am Hamburger Beispiel gut illustrieren. Nachdem die Zuständigkeiten für die öffentlich-rechtlichen Gerichtszweige beim Justizsenator gebündelt worden waren, beantragte die oppositionelle CDU, die Reform fortzuführen und ihm nun auch die Arbeits- und Sozialgerichte zu unterstellen (Wassermann 1964, S. 257). Diese Debatte wurden die Arbeitsgerichte nie mehr los. In regelmäßigen Abständen gab es im Verlauf der 1970er und 1980er Jahre Initiativen im Bundesrat, die die vollständige Realisierung der Idee des Rechtspflegeministeriums forderten (Binkert/ Preis 1987, S. 408). Sah sich die Arbeitsgerichtsbarkeit in der Weimarer Republik mit fiskalischen Argumenten konfrontiert, wurde die Sonderstellung nun mit politisch-liberalen Argumenten (Freiheit des Bürgers im Angesicht des Staates und Gewaltenteilung zwischen Verwaltung und Justiz) infrage gestellt. Die Politik der SPD, politische Liberalisierung in der Gerichtsorganisation zu verankern, erhöhte den Druck, auch die wirtschaftliche Seite des Liberalismus zuzulassen.

\section{Arbeitsgerichtsbarkeit in der Berliner Republik: Zurück zur Justiz?}

Es war dann die christlich-liberale Koalition, die im Jahr 1990 einen Gesetzentwurf durchsetzte, der es den Bundesländern ermöglichen sollte, den Einfluss der Justizressorts zu erweitern. Nach dem Arbeitsgerichtsgesetz von 1953 war das Arbeitsministerium federführend bei Verwaltung und Dienstaufsicht der Arbeitsgerichtsbarkeit, wobei in verschiedenen Angelegenheiten das Benehmen oder Einvernehmen mit den Justizbehörden gesucht werden musste. Das Arbeitsgerichtsgesetz-Änderungsgesetz (1990) eröffnete die Option, diese Zuständigkeiten umzukehren. Der neuerliche Liberalisierungsschub wurde einerseits mit dem Ziel begründet, den Bundesländern mehr Freiheitsspielräume zu gewähren. Liberalisierung bedeutet hier also auch, dass der Bund die Länder in die Freiheit entlässt - eine Entwicklung, die wir jüngst in verschiedenen Politikbereichen beobachten können. Vor allem aber stellte die Begründung des Gesetzes darauf ab, dass in vielen Ländern die Schaffung von Rechtspflegeministerien ja bereits begonnen habe und lediglich die Arbeitsgerichte noch inte- griert werden müssten (BT-Drucksache 11/5465).

Zu diesem Zeitpunkt war sehr unklar, welche Bundesländer von dem neuen Recht überhaupt Gebrauch machen würden, denn das Interesse daran hatte in den 1970er und 1980er Jahren stark variiert. Doch dann kam die deutsche Wiedervereinigung und mit ihr die Herausforderung, in den fünf neuen Ländern ein gänzlich neues Rechtssystem aufzubauen. Der Einigungsvertrag sah Übergangsregelungen für die Einrichtung der Sondergerichtsbarkeiten vor. Bis dahin sollte die arbeitsgerichtliche Rechtsprechung im Rahmen von Sonderkammern an den ordentlichen Gerichten ausgeübt werden (also die „Eingliederungslösung“) (Wassermann 1992, S. 265). Die eigenständigen Arbeitsgerichte wurden später aufgebaut, doch unterstellt wurden sie dem Justizressort (anfänglich mit Ausnahme von Brandenburg).

In Westdeutschland machte das sozialliberal regierte Rheinland-Pfalz als erstes Land von der Neuregelung Gebrauch. Heute sind Bayern und Berlin die einzigen Länder, in denen die Arbeitsgerichte noch beim Arbeitsressort angesiedelt sind - unabhängig von der parteipolitischen Regierungszusammensetzung. Von daher wundert es nicht, wenn das Arbeitsgerichtsbeschleunigungsgesetz aus dem Jahr 2000, mit dem die rot-grüne Regierung unter anderem die Einvernehmlichkeitsregelung kappte, weder in den Ausschussberatungen noch in den Parlamentsdebatten in diesem Punkt überhaupt kontrovers diskutiert wurde (BT-Drucksache 14/2490).

Die Begründungsmuster haben sich im Verlauf der 1990er Jahre wieder verschoben. Es treten zunehmend fiskalische Argumente in den Vordergrund, wodurch sich die Konfliktkonstellation der Weimarer Republik wiederholt. Das Arbeitsgerichtsbeschleunigungsgesetz soll dazu dienen, die Effizienz zu erhöhen. Flexibilitätsund Kostenargumente dominieren auch die neuerliche Runde in der Diskussion über Sondergerichte. Die Länder BadenWürttemberg und Sachsen haben eine Bundesratsinitiative gestartet, die es den Ländern ermöglichen soll, die öffentlichrechtlichen Gerichtsbarkeiten aus Kostengründen zusammenzulegen (BR-Drucksache 544/04). Die Justizministerkonferenz hat dieses Vorhaben auf ihrer Konferenz im Frühjahr 2005 unterstützt (Weth 2006, S. 182). Sollte dieses Vorhaben jemals realisiert werden, scheint mittelfristig auch das 
Schicksal der selbstständigen Arbeitsgerichte besiegelt.

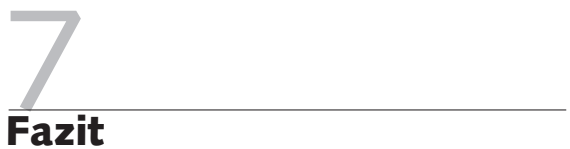

Schon historisch wurde die Sonderstellung der Arbeitsgerichte (und damit des Arbeitsrechts) außerhalb der Justiz mit der Besonderheit des Arbeitsvertrags und der Machtasymmetrie zwischen Arbeitnehmern und Arbeitgebern normativ begründet. Die Arbeiterklasse sollte über das Arbeitsrecht und die Arbeitsgerichte in den bürgerlichen Staat integriert werden. Die Anerkennung dieses Sonderstatus ist jedoch aufgrund zweier zentraler Faktoren in allen historischen Phasen stets prekär geblieben. Erstens: Die normativen Erwägungen sind auch bei den Sozialdemokraten immer dann in den Hintergrund gerückt, wenn fiskalische Gründe „einheitsrechtliche" Lösungen vorteilhaft erscheinen ließen. Zweitens: Ein Wertewandel im engeren Sinn hat sich vor allem bei der SPD vollzogen. Spätestens in den 1960er Jahren machte sie sich das rechtspolitische Liberalisierungsprojekt zu eigen. Damit wurde die Partei koalitionsfähig und attraktiv für die bürgerliche Mitte, die Beteiligungsund Freiheitsansprüche stellte. Gleichzeitig wurde es dadurch jedoch immer schwieriger, den Sonderstatus der Arbeitsgerichte noch zu rechtfertigen.

Es wird deutlich, welch ambivalentes Verständnis die deutsche Sozialdemokratie in ihrer Geschichte zum Verhältnis von Politik und Recht hatte. Hugo Sinzheimer sah die Chancen und Gefahren des politisierten Rechts. Er konnte nicht daran glauben, dass die konservative Justiz und die liberal denkenden Anhänger des bürgerlichen Rechts, das den Klassenbezug schon im Namen zu tragen schien, die Grundannahmen des Arbeitsrechts akzeptieren würden. Darum wollte er das Arbeitsrecht gegen ihren Einfluss abschirmen. Die SPD der 1960er Jahre hat offenkundig völlig anders gedacht. Sie hat an die Möglichkeit der Trennung von Exekutive und Judikative geglaubt, als sei das Justizministerium nicht Teil der Exekutive und als wären Gerichte nur dann politisch, wenn sie dem Einfluss der Verwaltung unterliegen. Die Idee der scheinbaren De-Politisierung des Rechts, institutionell ausgedrückt in einem Rechtspflegeministerium, leistete der ohnehin nie verstummten Kritik am Status der Arbeitsgerichtsbarkeit weiter Vorschub. Damit haben die Sozialdemokraten die Rechtfertigungsnot ,ihres“ Gerichtszweiges maßgeblich verstärkt. 
Bähr, J. (1991): Entstehung und Folgen des Arbeitsgerichtsgesetzes von 1926. Zum Verhältnis von Arbeiterschaft, Arbeiterbewegung und Justiz zwischen Kaiserreich und Nationalsozialismus, in: Tenfelde, K. (Hrsg.): Arbeiter im 20. Jahrhundert, Stuttgart, S. 507-532

Binkert, G./Preis, B. (1987): Subjekt und Objekt in der Arbeitsgerichtsbarkeit, in: Arbeit und Recht 12, S. 402-408

Bispinck, R. (2004): Kontrollierte Dezentralisierung der Tarifpolitik - eine schwierige Balance, in: WSI-Mitteilungen 5, S. 237-245

Blanke, T./Erd, R./Mückenberger, U./Stascheit, U. (Hrsg.) (1975): Kollektives Arbeitsrecht. Quellentexte zur Geschichte des Arbeitsrechts in Deutschland. Band 1: 1840-1933, Reinbek/Hamburg

Bohle, T. (1990): Einheitliches Arbeitsrecht in der Weimarer Republik. Bemühungen um ein deutsches Arbeitsgesetzbuch, Tübingen Bonner Kommentar zum Arbeitsrecht (1950), Bd. 10, Heidelberg, S. 41103

Brandt, W. (1979 [1969]): Regierungserklärung vom 28. Oktober 1969, in: Die großen Regierungserklärungen der deutschen Bundeskanzler von Adenauer bis Schmidt, München/Wien, S. 251-281

Graf, G. (1993): Das Arbeitsgerichtsgesetz von 1926, Goldbach

Kern, E. (1961): Das neue Richtergesetz, in: Juristenzeitung 20,

S. 617-621

Kraushaar, B. (1987): Die Arbeitsgerichte zwischen Arbeits- und Justizministerium, in: Betriebs-Berater 34, S. 2309-2314

Leinemann, W. (1991): Die geschichtliche Entwicklung der Arbeitsgerichtsbarkeit bis zur Errichtung des BAG, in: Neue Zeitschrift für Arbeitsrecht 24, S. 961-967

Massa-Wirth, H./Seifert, H. (2004): Betriebliche Bündnisse für Arbeit nur mit begrenzter Reichweite?, in: WSI-Mitteilungen 5, S. 246-254

Nautz, J. P. (1985): Die Durchsetzung der Tarifautonomie in Deutschland, Frankfurt/Main

Neumann, D. (1993): Kurze Geschichte der Arbeitsgerichtsbarkeit, in: Neue Zeitschrift für Arbeitsrecht 8, S. 342-345
Nikisch, A. (1961): Entschließung der Konferenz der Präsidenten der Landesarbeitsgerichte über die Ausbildung an den Universitäten im Arbeitsrecht, in: Juristenzeitung 5/6, S. 158

O'Brien, D. M. (1999): Institutional Norms and Supreme Court Opinions: On Reconsidering the Rise of Individual Opinions, in: Clayton, C. W./Gillman, H. (Eds.): Supreme Court Decision-Making. An Institutionalist Perspective, Chicago/London, pp. 91-114

Opolony, B. (2004): 25 Jahre ArbGG 1979 - Ein Blick zurück nach vorn, in: Neue Zeitschrift für Arbeitsrecht 10, S. 519-524

Rehder, B. (2003): Betriebliche Bündnisse für Arbeit in Deutschland. Mitbestimmung und Flächentarif im Wandel, Frankfurt/Main

Rehder, B. (2006): Recht und Politik beim Wandel des deutschen Flächentarifs. Juristen als politische Akteure im System der Arbeitsbeziehungen, in: Politische Vierteljahresschrift 2, S. 169-192

Rüthers, B. (1968): Die unbegrenzte Auslegung. Zum Wandel der Privatrechtsordnung im Nationalsozialismus, Tübingen

Sinzheimer, H. (1976 [1924]): Der Kampf um das neue Arbeitsrecht, in: Arbeitsrecht und Rechtssoziologie. Gesammelte Aufsätze und Reden; Kahn-Freund, O./Ramm, T. (Hrsg.), Band 1, Frankfurt, S. 91-99 Steidle, T. (1946): Der Plan für den Aufbau des Rechtspflegewesens in der amerikanischen Zone, in: Süddeutsche Juristen-Zeitung 1, S. 13-15 Wassermann, R. (1964): Annäherung der Gerichtsbarkeiten?, in: Juristische Rundschau, S. 256-258

Wassermann, R. (1992): Die Vereinheitlichung des Rechtswesens. Von der sozialistischen Rechtspflege zur Justiz des freiheitlich-demokratischen Rechtsstaats, in: Jesse, E./Mitter, A. (Hrsg.): Die Gestaltung der deutschen Einheit. Geschichte - Politik - Gesellschaft, Bonn/Berlin, S. 246-278 Weiß, J. (1994): Arbeitsgerichtsbarkeit und Arbeitsgerichtsverband im Kaiserreich und in der Weimarer Republik, Frankfurt/Main Weth, S. (2006): Einige prozessrechtliche Anmerkungen zur großen Justizreform, in: Neue Zeitschrift für Arbeitsrecht 4, S. 182-187 